\title{
Mechanism and New Findings in Brugada Syndrome
}

\author{
Wataru Shimizu, MD; Takeshi Aiba, MD; Shiro Kamakura, MD
}

\begin{abstract}
Brugada syndrome is a clinical entity characterized by coved type ST-segment elevation in the right precordial electrocardiographic leads ( $\left.\mathrm{V}_{1-3}\right)$ and an episode of ventricular fibrillation in the absence of structural heart disease. Although a number of clinical and experimental reports have elucidated the electrocardiographic, electrophysiologic, cellular, and molecular aspects, several problems remain unsolved. Recently developed high-resolution optical mapping techniques in arterially-perfused wedge preparations enable recording of transmembrane action potentials from 256 sites simultaneously at the epicardial surface, thus providing further advances in the understanding of the cellular mechanism of the specific ST-segment elevation and subsequent ventricular arrhythmias. In this review article, new findings relating to several unresolved problems such as gender difference (male predominance) and ethnic difference (higher incidence in Asian population) are also presented. (Circ J 2007; Suppl A: A-32-A-39)
\end{abstract}

Key Words: Brugada syndrome; Ethnicity; Gender; Genetics; Mutation; Polymorphism; ST-segment; Ventricular fibrillation

B rugada syndrome (BS) is characterized by covedtype ST-segment elevation in the right precordial electrocardiography (ECG) leads ( $\left.\mathrm{V}_{1-3}\right)$ and an episode of ventricular fibrillation (VF) in the absence of acute ischemia, electrolyte abnormalities or structural heart disease ${ }^{1-8}$ A type-1 ST-segment elevation, which is defined as a coved ST-segment elevation of $\geq 0.2 \mathrm{mV}$ at the $\mathrm{J}$ point with or without a terminal negative $\mathrm{T}$ wave, is required to diagnose BS, regardless of the absence or presence of sodium-channel blockers (Figs 1A,B)? A type-1 ST-segment elevation recorded only in the higher $\mathrm{V}_{1-2}$ leads (ie, $3^{\text {rd }}$ and $2^{\text {nd }}$ intercostal spaces) has been suggested to show similar prognostic value for subsequent cardiac events as that recorded in the standard $\mathrm{V}_{1-2}$ leads (Fig 1C) $)^{7,9,10} \mathrm{~A}$ type-2 saddle-back ST-segment elevation alone is not diagnostic for BS (Fig 1B). The prevalence of this syndrome is estimated to be 5 per 10,000 inhabitants, and is one of the important causes of sudden cardiac death of middle-aged males in Asian countries particularly!1,12 BS usually manifests during adulthood, with a mean age of sudden death of $41 \pm 15$ years, and child cases are rare? A family history of unexplained sudden death is present in approximately 20 $40 \%$ of the population in Western countries, and less (15$20 \%$ ) in Japan, $4,7,13,14$ A significant male predominance in BS has long been reported, and more than $80 \%$ of patients in Western countries and more than $90 \%$ of patients in Asian countries affected with BS are men ${ }^{15}$ Since Brugada and Brugada described 8 patients with a history of aborted sudden cardiac death caused by VF as a distinct clinical entity in 1992, a number of clinical and experimental reports from around the world have demonstrated the clinical, electrocardiographic, electrophysiologic, cellular, ionic, genetic and molecular features of $\mathrm{BS}^{2-14}$ However, several

(Received January 25, 2007; revised manuscript received February 7, 2007; accepted February 8, 2007)

Division of Cardiology, Department of Internal Medicine, National Cardiovascular Center, Suita, Japan

Mailing address: Wataru Shimizu, MD, Division of Cardiology, Department of Internal Medicine, National Cardiovascular Center, 5-7-1 Fujishiro-dai, Suita 565-8565 Japan. E-mail: wshimizu@hsp.ncvc. go.jp problems remain unsolved, such as genetic heterogeneity, late onset of first cardiac events, and gender and ethnic differences? In this review article, we present our recent data relating to the cellular and molecular mechanism of BS, the late onset of its clinical manifestation, male predominance, and higher incidence in Asian populations.

\section{Genetic and Molecular Aspects}

Advances in molecular genetics in the past decade have established a link between several inherited cardiac arrhythmias, including BS and long QT syndrome, and mutations in genes encoding ion channels, membrane components or receptors ${ }^{16}$ In 1998, the first mutation linked to BS was identified by Chen et al in $S C N 5 A{ }^{17}$ the gene encoding the a subunit of the sodium channel. Thereafter, a large family of BS was reported to link to a second locus on chromosome 3, which is close to but different from the SCN5A locus; ${ }^{18}$ however, specific gene or genes other than SCN5A have not yet been identified on chromosome 3. SCN5A mutations are reported to account for $18-30 \%$ of clinically diagnosed BS patients at present? Antzelevitch et al have recently reported that 3 probands associated with a BS-like ST-segment elevation and a short QT interval were linked to mutations in CACNAIC (A39V and G490R) or CACNB2 (S481L), the gene encoding the $\alpha 1$ or $\beta 2 b$ subunit of the L-type calcium channel, respectively! ${ }^{19}$ Their genetic and heterologous expression studies revealed loss of function of the L-type calcium channel current (ICa-L). However, approximately two-thirds of BS patients have not been yet genotyped, suggesting the presence of genetic heterogeneity? Other candidate genes for the Brugada phenotype include those encoding the transient outward current (Ito) and the delayed rectifier potassium current (ІК), or those coding the adrenergic receptors, cholinergic receptors, ion-channel-interacting protein, promoters, transcriptional factors, neurotransmitters, or transporters? 7,8

Among the approximately 100 mutations in SCN5A linked to BS, some of them have been studied in expression systems, and have been shown to result in loss of function of the sodium channel current ( $\mathrm{INa}$ ) by several mechanisms ${ }^{20}$ 
A Baseline

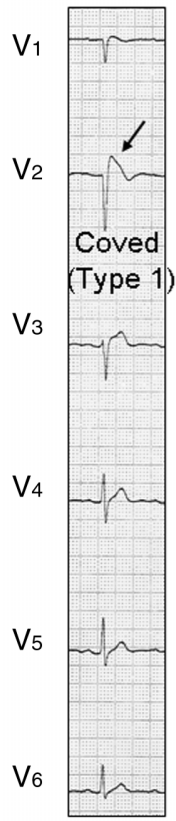

B

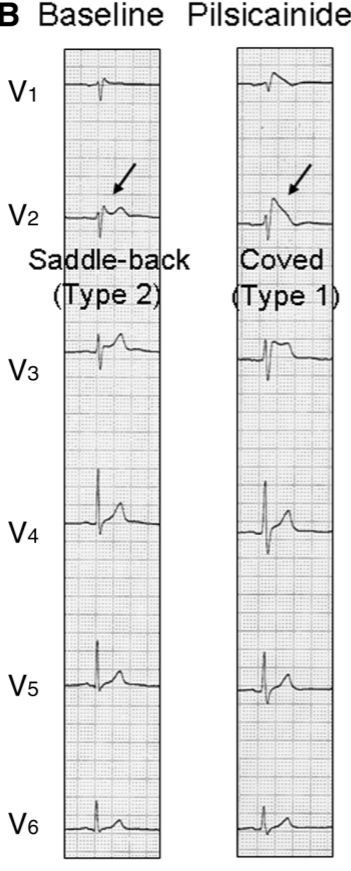

C Baseline Higher Inter-Costal Space

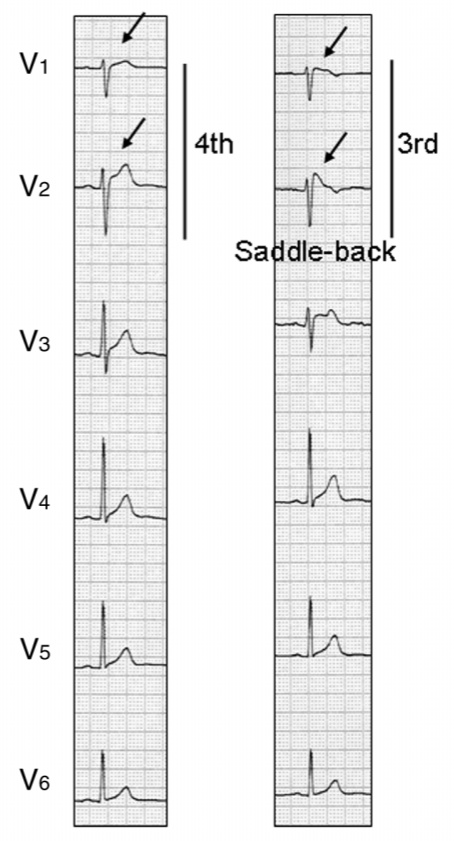

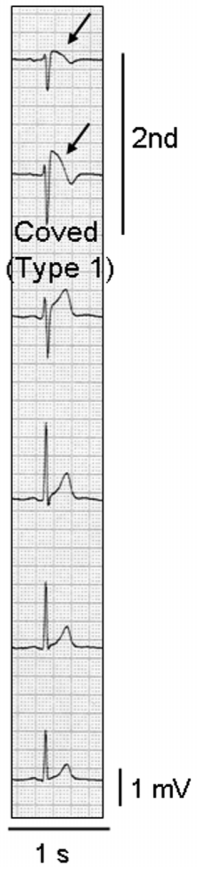

Fig 1. (A) Spontaneous type 1 coved type ST-segment elevation (arrow). (B) Unmasking of ST-segment elevation by a class IC sodium-channel blocker, pilsicainide. Under baseline conditions, type 2 saddle-back type ST-segment elevation is recorded in lead $\mathrm{V}_{2}$ (Left, arrow). Pilsicainide injection $(30 \mathrm{mg}$ ) unmasks the type 1 coved type ST-segment elevation in lead $\mathrm{V}_{2}$ (Right, arrow). (C) Unmasking of the type 1 electrocardiogram (ECG) by recording the right precordial $\left(\mathrm{V}_{1-2}\right)$ leads at the $3^{\text {rd }}$ and $2^{\text {nd }}$ intercostal spaces. No significant ST-segment elevation is observed in leads $V_{1}$ and $V_{2}$ of the standard 12-lead ECG (4th intercostal space) (Left, arrow), whereas saddle-back type (Middle, arrow) and type 1 coved type (Right, arrow) ST-segment elevation are unmasked in leads $V_{1}$ and $V_{2}$ recorded from the $3^{\text {rd }}$ and $2^{\text {nd }}$ intercostal spaces, respectively.

These functional effects include: (1) lack of expression of the sodium channel; (2) a shift in the voltage-dependence and time-dependence of INa activation, inactivation or reactivation; (3) entry of the sodium channel into an intermediate state of inactivation from which it recovers more slowly; (4) accelerated inactivation of the sodium channel; and (5) a trafficking defect. Some common SCN5A polymorphisms are reported to modulate the functional consequences of primary SCN5A mutations. Baroudi et al first suggested that the interaction of SCN5A polymorphisms and SCN5A mutations may affect the consequence of the functional effects. They reported that a common polymorphism (R1232W) of SCN5A affected protein trafficking when it was co-expressed with a T1620M mutation, although the T1620M mutation alone produced only gating abnormalities in the $\mathrm{INa}_{2}{ }^{21} \mathrm{On}$ the other hand, another common polymorphism (H558R) of SCN5A was reported by Ye et al to rescue normal trafficking and normal INa for the M1766L mutant protein?22 These effects of common SCN5A polymorphisms on modifying the functional consequence of $S C N 5 A$ mutations may make the clinical phenotype more complex.

\section{Cellular Mechanism of Brugada Phenotype}

The Ito-mediated phase 1 notch of the action potential (AP) has been reported to be larger in the epicardium than in the endocardium in many species, including humans ${ }^{23}$ Because the maintenance of the AP dome is determined by the fine balance of currents active at the end of phase 1 of the AP (principally Ito and ICa-L), any interventions that cause a net outward shift in the current active at the end of phase 1 can increase the magnitude of the AP notch, leading to loss of the AP dome (all-or-none repolarization) in the epicardium, but not in the endocardium, contributing to a significant voltage gradient across the ventricular wall during ventricular activation? 23 The heterogeneous loss of the AP dome in the epicardium has been shown to produce premature beats via a mechanism of phase 2 reentry in experimental studies using isolated sheets of canine right ventricle ${ }^{24}$ Therefore, these mechanism of all-or-none repolarization in the epicardial cells and phase 2 reentry-induced premature beat between the adjacent epicardial cells were expected to be responsible for the clinical phenotype in BS.

In the late 1990s, Antzelevitch's group developed an experimental model of BS using arterially perfused canine right ventricular (RV) wedge preparations, in which transmembrane APs and pseudo-ECGs were simultaneously recorded. These experimental studies have provided significant insights of the cellular mechanism of the Brugada phenotype, ST-segment elevation and subsequent VF 25,26 The Ito-mediated AP notch and the loss of the AP dome in the epicardial cells, but not in the endocardial cells, of the right ventricle gives rise to a transmural voltage gradient, producing ST-segment elevation in the ECG in the wedge preparations. Fig 2 shows transmembrane APs simultaneously recorded from 2 epicardial (Epi) and 1 endocardial sites, together with a transmural ECG in a Brugada model using the RV wedge preparation. Under control conditions, a small $\mathbf{J}$ wave coincides with the small notch observed in the epicardial cells, but not in the endocardial cells (Fig 2A). Combined administration of terfenadine (ICa-L block) and pilsicainide (INa block) produces a loss of the AP dome in 


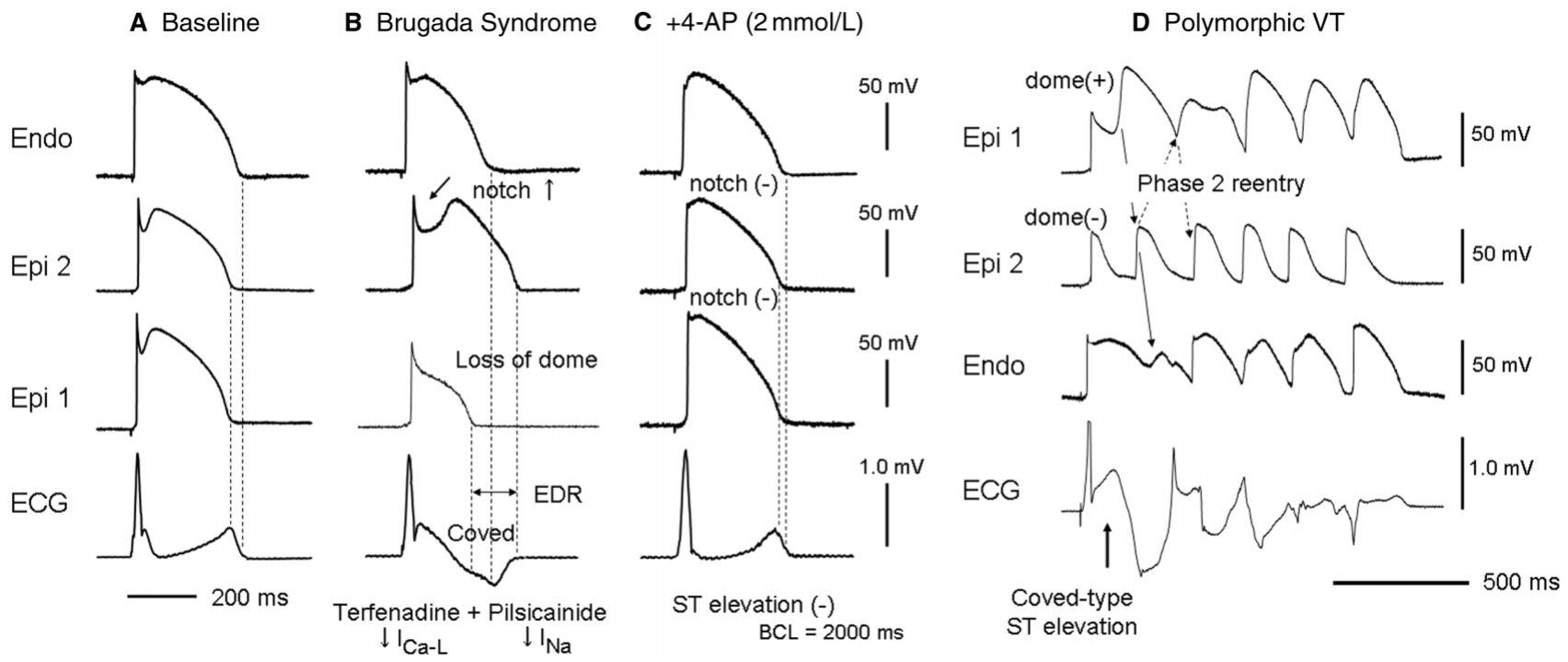

Fig 2. Type 1 coved type ST-segment elevation and non-sustained polymorphic ventricular tachycardia (VT) via phase 2 reentry induced in a Brugada model using an arterially perfused canine right ventricular wedge preparation. Shown are transmembrane action potentials (APs) simultaneously recorded from 2 epicardial sites (Epi 1 and Epi 2) and 1 endocardial site (Endo) together with a transmural ECG (basic cycle length $(\mathrm{BCL})=2,000 \mathrm{~ms}$ ). (A) Under baseline conditions, phase 1 AP notch in Epi, but not in Endo, is associated with a J wave in the ECG. (B) Combined administration of terfenadine $(5 \mu \mathrm{mol} / \mathrm{L})$ and pilsicainide $(5 \mu \mathrm{mol} / \mathrm{L})$ produces a loss of AP dome in Epi 1, but not in Epi 2, resulting in a marked epicardial dispersion of repolarization (EDR), and a coved-type ST segment elevation and a negative T wave in the ECG. (C) 4-aminopyridine (4-AP), a selective blocker of the transient outward current (Ito) (2 mmol/L), restores the AP dome, decreases the phase 1 AP notch, and normalizes the ST-segment elevation. (D) In the setting of heterogeneous loss of the AP dome (coexistence of loss of dome regions and restored dome regions) in the epicardium and a remarkable coved type ST-segment elevation in the ECG with combined administration with terfenadine and pilsicainide, electrotonic propagation from the site where the dome is restored (Epi 1) to the site where it is lost (Epi 2) results in development of a premature beat induced by phase 2 reentry, triggering spontaneous polymorphic VT (Modified from Nat Clin Pract Cardiovasc Med 2005; 2: 408-414 with permission).

Epi 1, but not in Epi 2, resulting in a marked epicardial dispersion of repolarization (EDR), and a coved-type ST segment elevation and negative $\mathrm{T}$ wave in the ECG (Fig 2B). A selective Ito blocker, 4-aminopyridine, restores the AP dome, decreases the phase 1 AP notch, and normalizes the ST-segment elevation (Fig 2C). Fig 2D shows non-sustained polymorphic ventricular tachycardia (VT) via phase 2 reentry induced in a Brugada model using the wedge preparation. In the setting of remarkable coved type ST-segment elevation with combined administration of terfenadine and pilsicainide, heterogeneous loss of the AP dome (coexistence of loss of dome regions and restored dome regions) in the epicardium creates a marked EDR, giving rise to premature beats caused by phase 2 reentry, which precipitates non-sustained polymorphic VT.

\section{Optical Mapping Study}

The AP data in the Brugada model using arterially perfused canine RV wedge preparations strongly supported the hypothesis that episodes of VF in BS are triggered by premature beats between the adjacent epicardial cells via the mechanism of phase 2 reentry. However, the precise mechanism of the initial premature beats and the maintenance of non-sustained polymorphic VT or VF remain unsolved, because the number of AP recording sites available for floating microelectrodes is small in the wedge preparations. To overcome this limitation, we recently developed high-resolution $(256 \times 256)$ optical mapping techniques that allowed us to record transmembrane APs from 256 sites simultaneously at the epicardial or endocardial surface of the wedge preparations (Figs 3-5), ,27 Fig 3 shows the mechanism of phase 2 reentry-induced premature beats (P2Rextrasystoles) under Brugada-ECG conditions. A steep repolarization gradient between the loss of dome region and the restored dome region in the epicardium, but not in the endocardium, develops the initial P2R-extrasystole. We then recorded spontaneous episodes of P2R-extrasystoles and subsequent non-sustained polymorphic VT or VF under these conditions, and analyzed the epicardial AP duration (APD) and conduction velocity (Figs 4,5). Once again, most of the P2R-extrasystoles originated from the area showing the steepest (maximum) gradient of repolarization (GRmax) between the loss of dome site and the restored dome site in the epicardium (Figs 4C,5C, arrows), leading to non-sustained polymorphic VT or VF. These data also indicate that a steep repolarization gradient between the loss of dome region and the restored dome region in the epicardium is essential to produce the P2R-extrasystoles that precipitate polymorphic VT or VF. On the other hand, the epicardial GRmax does not differ between episodes of polymorphic VT and those of VF. Figs 4D,E and 5D,E show the mechanism underlying the difference between polymorphic VT and VF. Just before inducing the episodes of polymorphic VT or VF, the epicardial depolarization map paced from the endocardium at the basic cycle length of $2,000 \mathrm{~ms}$ shows a remarkable conduction delay in the episode of VF (Fig 5D) compared with that of polymorphic VT (Fig 4D). The conduction parameters, such as QRS duration and interval between the stimulus and the earliest epicardial activation, are significantly longer in the episodes of VF than in those of polymorphic VT. Figs 4A,B 

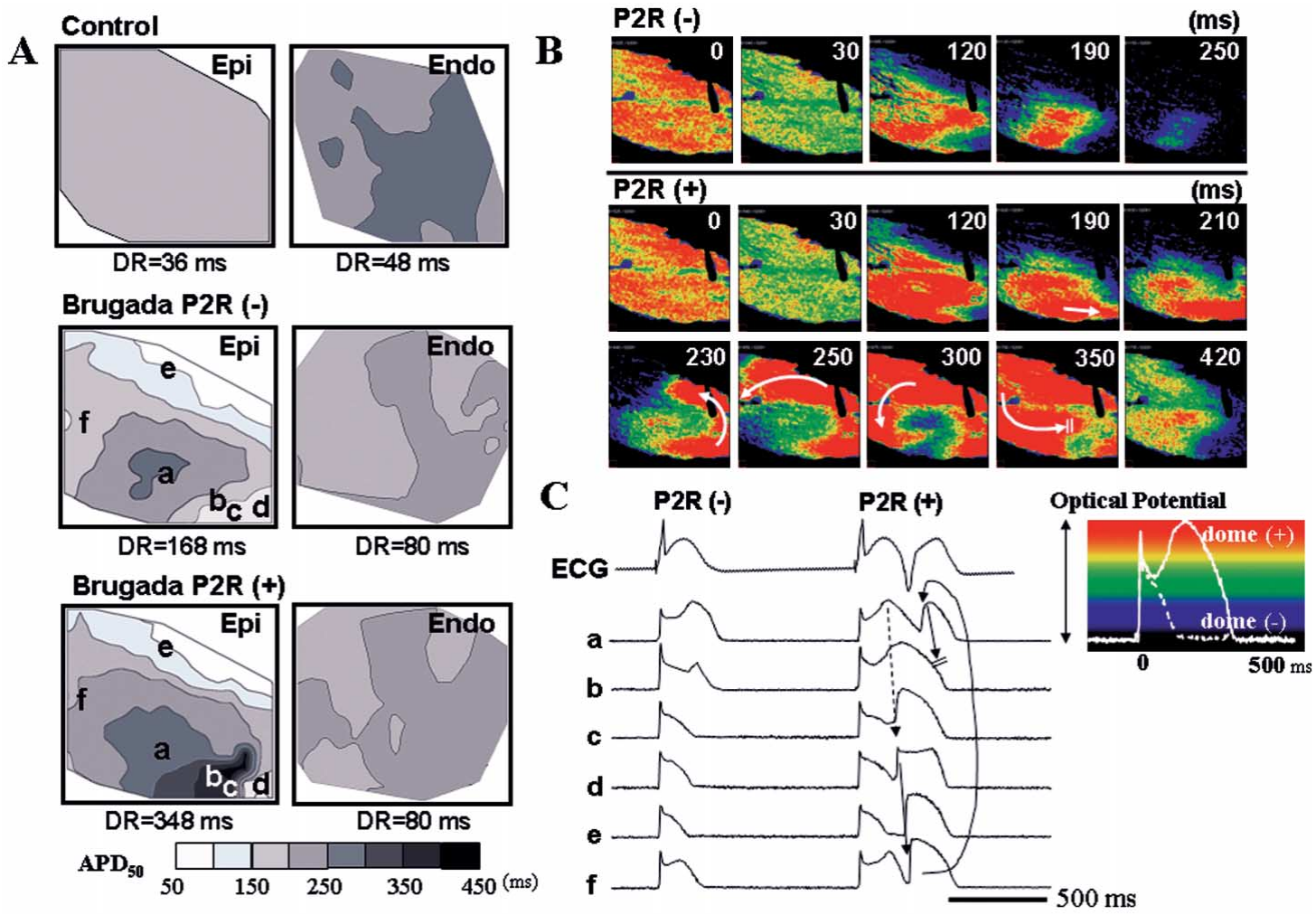

Fig 3. Mechanism of the phase 2 reentry-induced premature beats (P2R-extrasystoles) under the condition of BrugadaECG in a model using a wedge preparation combined with high-resolution $(256 \times 256)$ optical mapping techniques. (A) Representative action potential duration measured at 50\% (APD50) contour map on the right ventricular epicardium (Epi) and endocardium (Endo) in the control, in the ST-segment elevation (Brugada-ECG) without phase 2 reentrant extrasystoles (P2R (-)) and in the Brugada-ECG just before P2R extrasystoles (P2R (+)). (B) Snapshots of an optical isopotential movie on the Epi surface during P2R(-) and P2R(+) in the Brugada-ECG. (C) Optical action potentials (APs) at each site (a-f) on the Epi surface and transmural ECG. Under the Brugada-ECG, the AP morphology in Epi, but not Endo, changes to heterogeneous because of the combination of abbreviated (loss-of-dome; site d,e) and prolonged (restore-of-dome; site a,b) APs, resulting in increasing dispersion of repolarization (DR) in Epi (168 ms) rather than in Endo (80 ms). Further prolongation of the AP in the Epi area (site b) is closely adjacent to the loss-of-dome APs (site d), thus producing a repolarization mismatch within a small area $(\mathrm{DR}=348 \mathrm{~ms})$ and developing a P2R-extrasystole at the loss-of-dome site (site d). Thus, a steep repolarization gradient in Epi, but not in Endo, develops the initial P2R-extrasystole in the Brugada-ECG (Modified from J Am Coll Cardiol 2006; 47: 2074-2085 with permission).

represents a phase map and the optical APs during the P2Rinduced polymorphic VT, showing that reentry is initiated from the epicardial GRmax area and rotates mainly in the epicardium without wave-break. In contrast, Figs 5A,B represents these during P2R-induced VF, showing that the development of the initial P2R is similar to that of polymorphic VT, but that the first P2R-wave is broken up into multiple wavelets, resulting in degeneration of VT into VF. The phase singularity points during the first P2R-wave almost coincide with the sites of delayed conduction (Fig 5D). Wave-break during the first P2R-extrasystole produces multiple wavelets in the episodes of $\mathrm{VF}$, whereas no wavebreak or wave-break followed by wave collision and termination occurs in the episodes of polymorphic VT. Figs 4E and $5 \mathrm{E}$ are histograms of the epicardial APD measured at 50\% (APD50) during the first P2R-wave. There is a large variety of APD50 in the epicardium during the first P2Rwave in the episodes of VF, whereas only slight variety in the APD50 is observed in the episodes of polymorphic VT. These data suggest that both conduction delay and dispersion of repolarization play significant roles in the perpetuation of VF episodes.

\section{Late Onset of Clinical Manifestation}

Because BS is a primary electrical disease, and at least one-third of the patients have mutations in ion channel genes (SCN5A, CACNA1C, CACNB2), clinical manifestation during childhood would be expected. However, BS usually manifests in middle age, at 40-50 years of age? Frustaci et al recently reported a significant myocytes apotosis in both the right and left ventricular myocardium in a histological study of BS patients with SCN5A mutations, and suggested that abnormal function of the sodium channels may lead to a sufficient degree of cellular damage, attributing to the arrhythmic event ${ }^{2} 8$ We recently analyzed several ECG parameters recorded during long-term follow-up of BS patients with and without the SCN5A mutation ${ }^{29}$ In both patient groups, the depolarization parameters, including $\mathrm{P}$ wave, QRS, S wave duration and PQ interval, increased with age, especially in patients with the SCN5A mutation. Taken together with the experimental data ${ }^{27}$ the findings suggest that depolarization abnormalities (conduction slowing) are required for the maintenance of VF in BS, although the initiating premature beats are caused by a phase 2 reentry mechanism. 

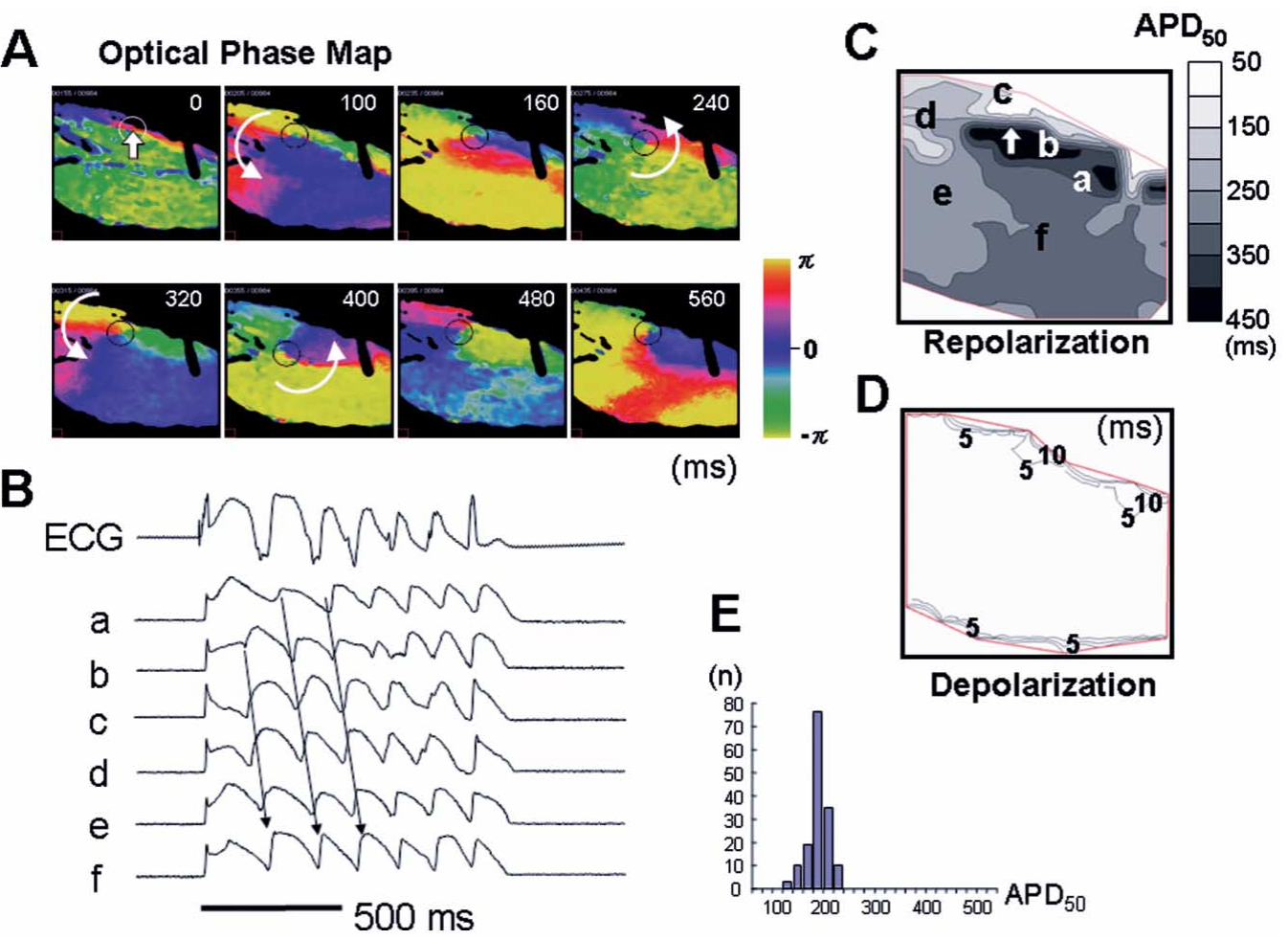

Fig 4. Mechanism underlying non-sustained polymorphic ventricular tachycardia (VT) in a Brugada model using a wedge preparation combined with high-resolution $(256 \times 256)$ optical mapping techniques. (A) Representative snapshots from a phase movie during polymorphic VT originating from epicardial (Epi) phase 2 reentry (P2R). (B) Optical action potentials at each site $(a-f)$, together with a transmural ECG. (C, D) Repolarization and depolarization maps on the Epi surface in the condition of Brugada-ECG just before polymorphic VT. (E) Epi action potential duration at 50\% repolarization (APD50) histogram during the first P2R-wave. Reentry is initiated from the steepest (maximum) repolarization gradient site in Epi (arrow in A and C) and rotates mainly in Epi without wave-break. The Epi depolarization map paced from Endo shows no conduction delay (D). There is a little variety of APD in Epi during the first P2R-wave (E). Open circles mark phase singularity points (Modified from J Am Coll Cardiol 2006; 47: 2074-2085 with permission).

\section{Male Predominance}

Because all mutations so far identified in SCN5A display an autosomal dominant mode of transmission in BS, males and females would be expected to inherit the defective gene equally. However, an apparent male predominance is observed in patients with BS $!^{15}$ Di Diego et al suggested the cellular basis for male predominance in BS while using arterially-perfused canine RV wedge preparations ${ }^{30}$ They reported that the Ito-mediated phase $1 \mathrm{AP}$ notch in the RV epicardium was larger in male dogs than in female dogs was responsible for the male predominance in the Brugada phenotype. On the other hand, the male hormone, testosterone, has been reported to increase the outward potassium currents (the rapidly $[\mathrm{IKr}]^{31,32}$ and the slowly $[\mathrm{IKs}]^{33}$ activating component of Ік, and the inward rectifier potassium current $\left[\mathrm{IK}_{1}\right]^{32}$ ) or decrease the inward currents (ICa-L) ${ }^{33}$ Therefore, testosterone would be expected to accentuate the Brugada phenotype. Clinically, Matsuo et al report 2 cases of asymptomatic BS in which typical coved ST-segment elevation disappeared following orchiectomy as therapy for prostate cancer ${ }^{34}$ supporting the expectation for testosterone. Moreover, testosterone is also known to decrease visceral fat ${ }^{35}$ and patients with BS are thinner than the normal population 36 On the basis of these clinical and experimental findings, we directly measured the testosterone level in male patients with BS and compared them with age-matched normal males? ${ }^{37}$ The testosterone level was significantly higher and body mass index (BMI) significantly lower in the Brugada males than in the controls after adjusting for several confounding variables influencing testosterone level or BMI (eg, age, exercise, stress, smoking, and medication). Interestingly, testosterone level was inversely correlated with BMI in both Brugada and control males even after adjusting for confounding variables, suggesting that Brugada males have a higher testosterone level associated with lower visceral fat (Fig 6). Moreover, conditional logistic regression model analysis showed that both higher testosterone level and lower BMI independently increase the risk of BS. These data suggest that the male predominance in the Brugada phenotype is at least in part related to testosterone, which is present only in males.

\section{Higher Incidence in Asian Population}

The incidence of BS is higher in Asian countries, including Thailand and Japan, than in Western countries: $11,12,38$ It has been reported that common polymorphisms might modulate the activity of the primary disease-causing mutation or influence susceptibility to arrhythmia, even in the general population 39 The common polymorphisms may attribute to ethnic differences in the clinical phenotype in inherited cardiac arrhythmias, including BS, because some common polymorphisms are ethnically dependent. Pfeufer et al reported that polymorphisms in the SCN5A promoter were associated with a widening of QRS duration in a cen- 
A Optical Phase Map
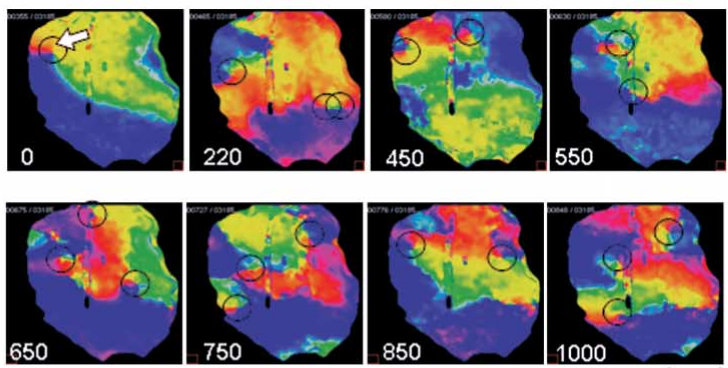

$(\mathrm{ms})$

B
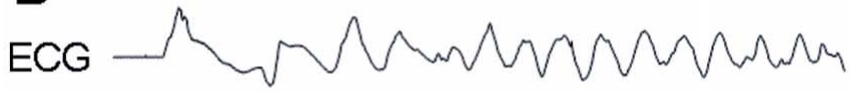

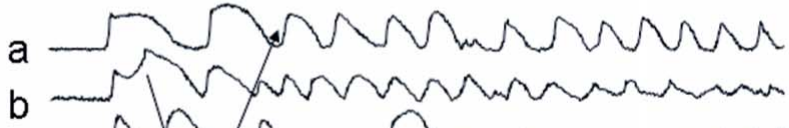

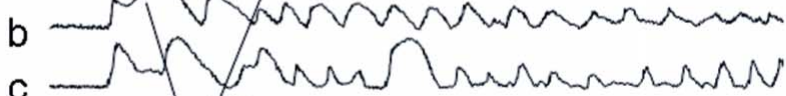

C
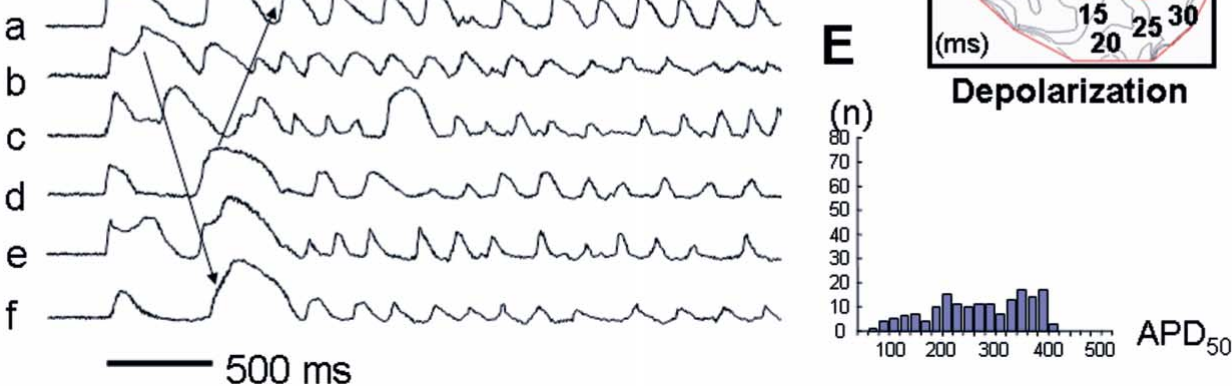

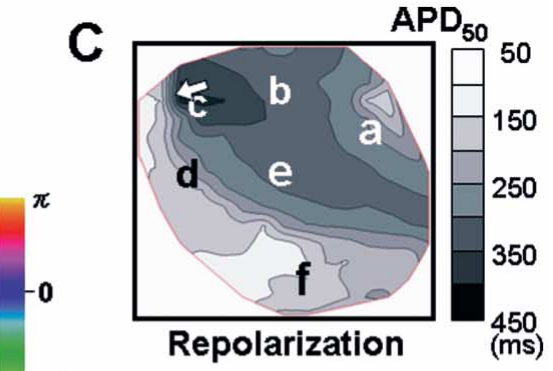

$\pi \mathbf{D}$

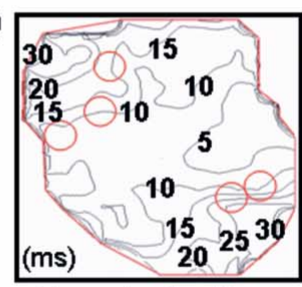

Fig 5. Mechanism underlying ventricular fibrillation (VF) in a Brugada model using a wedge preparation combined with high-resolution $(256 \times 256)$ optical mapping techniques. (A) Representative snapshots from a phase movie during VF originating from the epicardial (Epi) phase 2 reentry (P2R). (B) Optical action potentials at each site (a-f), together with a transmural ECG. (C, D) Repolarization and depolarization maps on the Epi surface in the condition of Brugada-ECG just before VF. (E) Epi action potential duration at 50\% repolarization (APD50) histogram during the first P2R-wave. The area of maximum gradient of repolarization in Epi (arrow in A and C) develops the P2R. The first P2R-wave is broken up into multiple wavelets (A, $220 \mathrm{~ms}$ ), resulting in degeneration of ventricular tachycardia into VF. The Epi depolarization map paced from the endocardium shows a remarkable conduction delay in the episode of VF (D). The phase singularity points during the first P2R-wave (open circle in D) almost coincide with the Epi sites of delayed conduction. There is a large variety of APD in Epi during the first P2R-wave (E). Thus, P2R-extrasystoles degenerate into VF with further depolarization and repolarization disturbances. Open circles mark phase singularity points (Modified from J Am Coll Cardiol 2006; 47: 2074-2085 with permission).
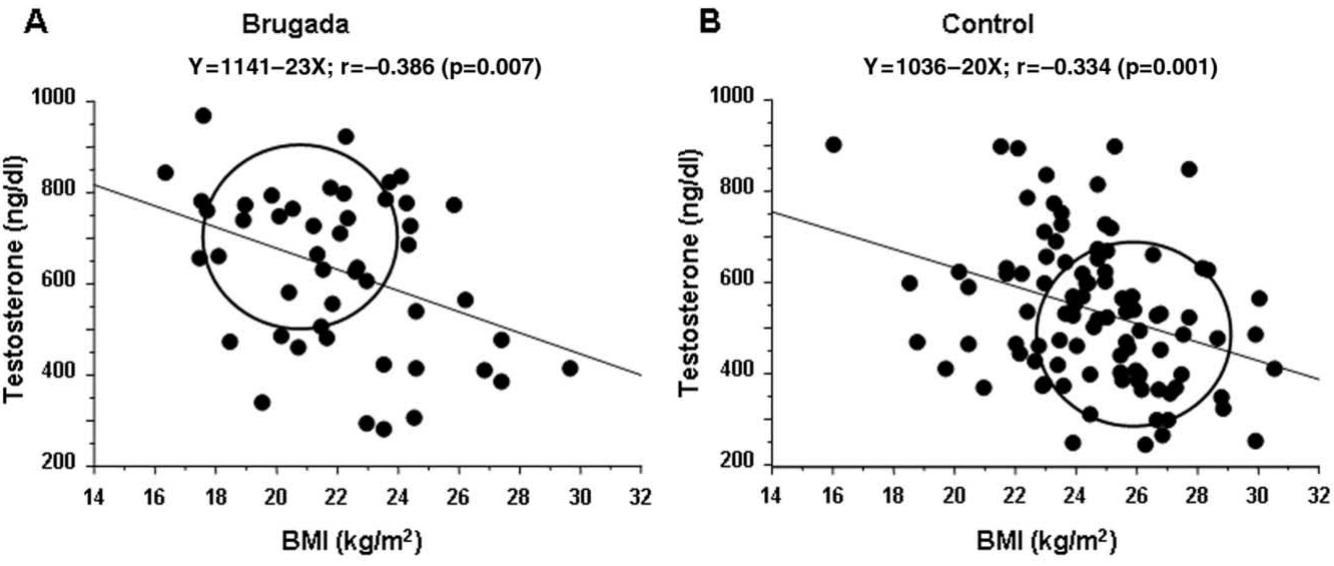

Fig 6. Correlation between testosterone level and body mass index (BMI) in Brugada syndrome males and age-matched control males. Testosterone level inversely correlated withe BMI in both groups (J Cardiovasc Electrophysiol 2007 (in press). with permission).

tral European general population. ${ }^{40}$ We recently identified a haplotype variant consisting of 6 individual DNA polymorphisms in near-complete linkage disequilibrium within the proximal promoter region of SCN5A in Asians only (an allele frequency of 22\%), not in Caucasian or African-
Americans (Fig 7) ${ }^{41}$ Luciferase reporter activity of this variant haplotype, designated Haplotype B, in cardiomyocytes is reduced $62 \%$ compared with the wild-type, designated Haplotype A. To test the hypothesis that this SCN5A promoter polymorphism may modulate variability in cardiac 


\section{Haplotype A Haplotype B Haplotype C}

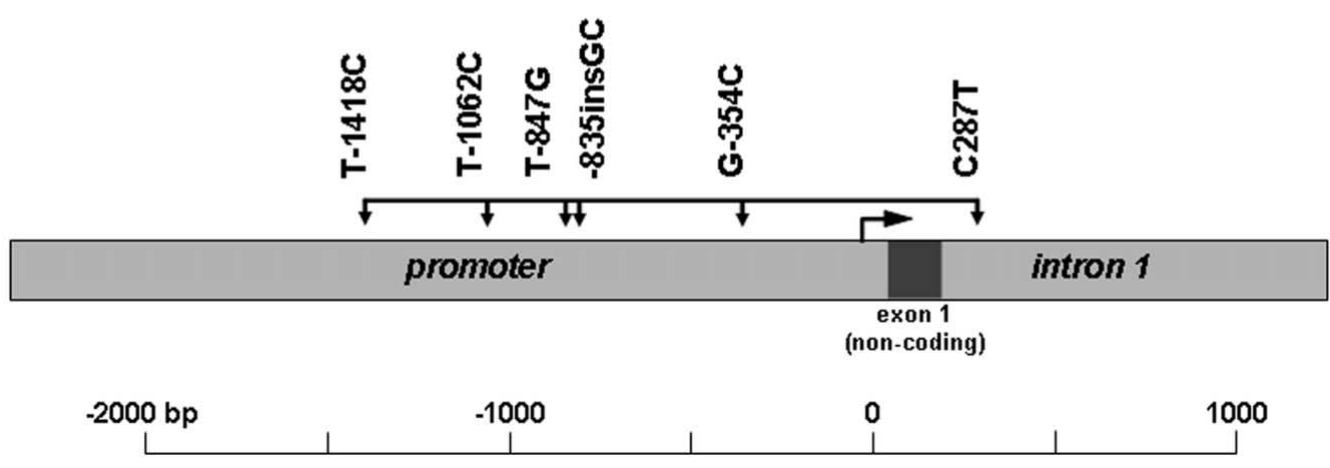

Fig 7. Haplotypes identified within the proximal promoter region of SCN5A, a cardiac sodium-channel gene. The 6 polymorphisms are in near-complete linkage disequilibrium. Haplotype A is designated as containing all common alleles, and Haplotype B as containing all minor alleles. The discordant haplotype is designated Haplotype C. *Frequency in the Japanese (control) population (Modified from Circulation 2006; 113: 338-344 with permission).

A

Brugada

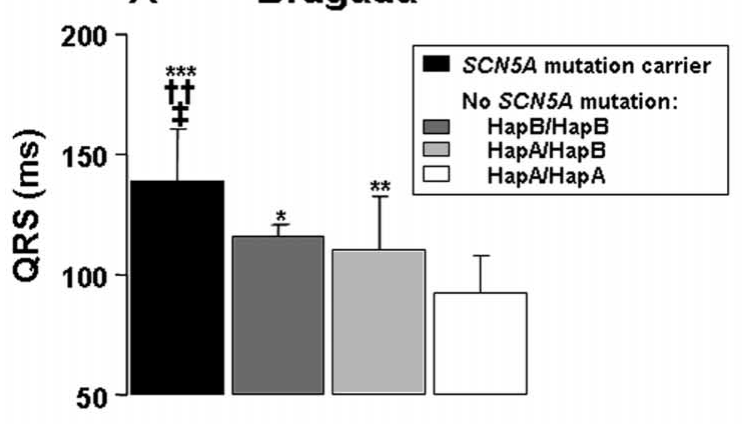

B Control

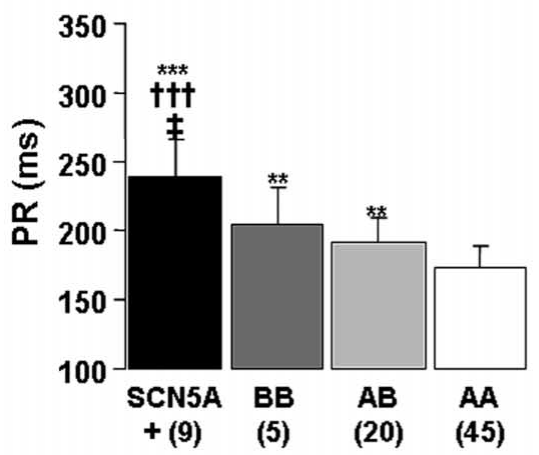

${ }^{*}$ Frequency

$75.5 \%$

$24 \%$

$0.5 \%$
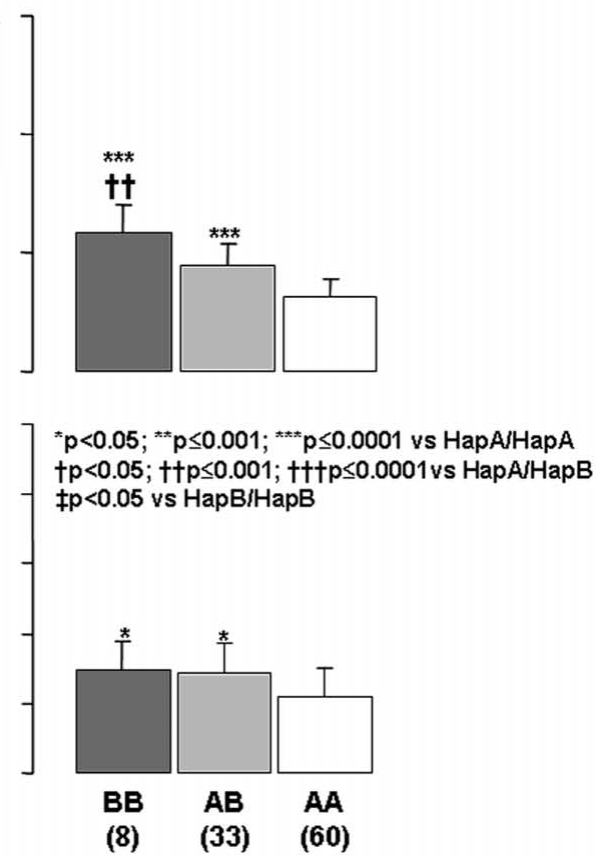

Fig 8. SCN5A promoter haplotype pair effects on QRS duration in lead $\mathrm{V}_{6}$ and PR duration in lead II in patients with Brugada syndrome and in control subjects. In the Brugada patients without SCN5A mutations and in the control subjects, both QRS and PR duration show a gene-dose effect, being longest in Haplotype B homozygotes (BB), intermediate in Haplotype A/Haplotype B heterozygotes (AB) and shortest in Haplotype A homozygotes (AA). The Brugada patients with SCN5A mutations show the longer duration of both QRS and PR than do those without SCN5A mutations. Patient numbers are indicated between parentheses. Data mean \pm SD (Modified from Circulation 2006; 113: 338 - 344 with permission).

conduction, the relationship between the $S C N 5 A$ promoter haplotype and indices of conduction velocity (ie, PR and QRS durations) was analyzed in a cohort of 71 Japanese BS subjects without SCN5A mutations and in 102 Japanese controls. In both groups, PR and QRS durations were significantly longer in Haplotype B individuals, with a genedose effect (Fig 8). Moreover, increases in both the PR and
QRS duration with sodium channel blockers, which are known to be arrhythmogenic in BS, were genotype-dependent and a gene-dose effect was also observed. These data demonstrate that the Haplotype B within the SCN5A promoter region alone does not give rise to BS, but that it likely contributes to a higher incidence of BS in Asian population in combination with other yet unknown (genetic) factors. 


\section{Acknowledgments}

Dr W Shimizu was supported by the Uehara Memorial Foundation, the Hoansha Research Foundation, Japan Research Foundation for Clinical Pharmacology, Ministry of Education, Culture, Sports, Science and Technology Leading Project for Biosimulation, and Health Sciences Research Grants (H18-Research on Human Genome-002) from the Ministry of Health, Labour and Welfare, Japan.

\section{References}

1. Brugada P, Brugada J. Right bundle branch block, persistent ST segment elevation and sudden cardiac death: A distinct clinical and electrocardiographic syndrome: A multicenter report. J Am Coll Cardiol 1992; 20: 1391-1396.

2. Alings M, Wilde A. "Brugada" syndrome: Clinical data and suggested pathophysiological mechanism. Circulation 1999; 99: 666-673.

3. Antzelevitch C, Brugada P, Brugada J, Brugada R, Shimizu W, Gussak I, et al. Brugada syndrome: A decade of progress. Circ Res 2002; 91: $1114-1118$.

4. Priori SG, Napolitano C, Gasparini M, Pappone C, Della Bella P, Giordano U, et al. Natural history of Brugada syndrome: Insights for risk stratification and management. Circulation 2002; 105: 1342 1347.

5. Wilde AA, Antzelevitch C, Borggrefe M, Brugada J, Brugada R, Brugada P, et al. Proposed diagnostic criteria for the Brugada syndrome: Consensus report. Circulation 2002; 106: 2514-2519.

6. Brugada J, Brugada R, Brugada P. Determinants of sudden cardiac death in individuals with the electrocardiographic pattern of Brugada syndrome and no previous cardiac arrest. Circulation 2003; 108: 3092-3096.

7. Antzelevitch C, Brugada P, Borggrefe M, Brugada J, Brugada R, Corrado D, et al. Brugada Syndrome: Report of the Second Consensus Conference: Endorsed by the Heart Rhythm Society and the European Heart Rhythm Association. Circulation 2005; 111: 659670.

8. Shimizu W, Aiba T, Kamakura S. Mechanisms of disease: Current understanding and future challenges in Brugada syndrome. Nat Clin Pract Cardiovasc Med 2005; 2: 408-414.

9. Shimizu W, Matsuo K, Takagi M, Tanabe Y, Aiba T, Taguchi A, et al. Body surface distribution and response to drugs of ST segment elevation in the Brugada syndrome: Clinical implication of 87-leads body surface potential mapping and its application to 12-leads electrocardiograms. J Cardiovasc Electrophysiol 2000; 11: 396-404.

10. Miyamoto K, Yokokawa M, Tanaka K, Nagai T, Okamura H, Noda $\mathrm{T}$, et al. Diagnostic and prognostic value of type 1 Brugada electrocardiogram at higher (third or second) V1 to V2 recording in men with Brugada syndrome. Am J Cardiol 2007; 99: 53-57.

11. Nademanee K, Veerakul G, Nimmannit S, Chaowakul V, Bhuripanyo $\mathrm{K}$, Likittanasombat K, et al. Arrhythmogenic marker for the sudden unexplained death syndrome in Thai men. Circulation 1997; 96: $2595-2600$

12. Atarashi H, Ogawa S, Harumi K, Sugimoto T, Inoue H, Murayama $\mathrm{M}$, et al. Three-year follow-up of patients with right bundle branch block and ST segment elevation in the right precordial leads: Japanese Registry of Brugada Syndrome: Idiopathic Ventricular Fibrillation Investigators. J Am Coll Cardiol 2001; 37: 1916-1920.

13. Kanda M, Shimizu W, Matsuo K, Nagaya N, Taguchi A, Suyama K, et al. Electrophysiologic characteristics and implication of induced ventricular fibrillation in symptomatic patients with Brugada syndrome. J Am Coll Cardiol 2002; 39: 1799-1805.

14. Eckardt L, Probst V, Smits JP, Bahr ES, Wolpert C, Schimpf R, et al. Long-term rognosis of individuals with right precordial ST-segmentelevation Brugada syndrome. Circulation 2005; 111: 257-263.

15. Shimizu W. Gender difference and drug challenge in Brugada syndrome (Editorial Comment). J Cardiovasc Electrophysiol 2004; 15: $70-71$.

16. Shimizu W. The long QT syndrome: Therapeutic implications of a genetic diagnosis. Cardiovasc Res 2005; 67: 347-356.

17. Chen Q, Kirsch GE, Zhang D, Brugada R, Brugada J, Brugada P, et al. Genetic basis and molecular mechanisms for idiopathic ventricular fibrillation. Nature 1998; 392: 293-296.

18. Weiss R, Barmada MM, Nguyen T, Seibel JS, Cavlovich D, Kornblit $\mathrm{CA}$, et al. Clinical and molecular heterogeneity in the Brugada syndrome: A novel gene locus on chromosome 3. Circulation 2002; 105: $707-713$.

19. Antzelevitch C, Pollevick GD, Cordeiro JM, Casis O, Sanguinetti MC, Aizawa Y, et al. Loss-of-function mutations in the cardiac calcium channel underlie a new clinical entity characterized by ST- segment elevation, short QT intervals, and sudden cardiac death. Circulation 2007; 115: 442-449.

20. Tan HL, Bezzina CR, Smits JP, Verkerk AO, Wilde AA. Genetic control of sodium channel function. Cardiovasc Res 2003; 57: 961 973.

21. Baroudi G, Acharfi S, Larouche C, Chahine M. Expression and intracellular localization of an SCN5A double mutant R1232W/T1620M implicated in Brugada syndrome. Circ Res 2002; 90: E11-E16.

22. Ye B, Valdivia CR, Ackerman MJ, Makielski JC. A common human SCN5A polymorphism modifies expression of an arrhythmia causing mutation. Physiol Genomics 2003; 12: 187-193.

23. Litovsky SH, Antzelevitch C. Transient outward current prominent in canine ventricular epicardium but not endocardium. Circ Res 1988; 62: $116-126$.

24. Krishnan SC, Antzelevitch C. Flecainide-induced arrhythmia in canine ventricular epicardium: Phase 2 Reentry? Circulation 1993; 87: $562-5729$

25. Yan GX, Antzelevitch C. Cellular basis for the electrocardiographic J wave. Circulation 1996; 93: 372-379.

26. Yan GX, Antzelevitch C. Cellular basis for the Brugada syndrome and other mechanisms of arrhythmogenesis associated with ST segment elevation. Circulation 1999; 100: 1660-1666.

27. Aiba T, Shimizu W, Hidaka I, Uemura K, Noda T, Zheng C, et al. Cellular basis for trigger and maintenance of ventricular fibrillation in the Brugada syndrome model: High resolution optical mapping study. J Am Coll Cardiol 2006; 47: 2074-2085.

28. Frustaci A, Priori SG, Pieroni M, Chimenti C, Napolitano C, Rivolta I, et al. Cardiac histological substrate in patients with clinical phenotype of Brugada syndrome. Circulation 2005; 112: 3680-3687.

29. Yokokawa M, Kitamura S, Okamura H, Noda T, Suyama K, Kurita $\mathrm{T}$, et al. Long-term follow-up of electrocardiographic features in patients with Brugada syndrome: Comparison between SCN5A mutation carriers and non-mutation carriers (abstract). Circulation 2006; 114: II-471.

30. Di Diego JM, Cordeiro JM, Goodrow RJ, Fish JM, Zygmunt AC, Perez GJ, et al. Ionic and cellular basis for the predominance of the Brugada syndrome phenotype in males. Circulation 2002; 106: 2004-2011.

31. Shuba YM, Degtiar VE, Osipenko VN, Naidenov VG, Woosley RL. Testosterone-mediated modulation of HERG blockade by proarrhythmic agents. Biochem Pharmacol 2001; 62: 41 -49.

32. Liu XK, Katchman A, Whitfield BH, Wan G, Janowski EM, Woosley $\mathrm{RL}$, et al. In vivo androgen treatment shortens the QT interval and increases the densities of inward and delayed rectifier potassium currents in orchiectomized male rabbits. Cardiovasc Res 2003; 57: $28-36$.

33. Bai CX, Kurokawa J, Tamagawa M, Nakaya H, Furukawa T. Nontranscriptional regulation of cardiac repolarization currents by testosterone. Circulation 2005; 112: 1701-1710.

34. Matsuo K, Akahoshi M, Seto S, Yano K. Disappearance of the Brugada-type electrocardiogram after surgical castration: A role for testosterone and an explanation for the male preponderance. Pacing Clin Electrophysiol 2003; 26: 1551-1553.

35. Glass AR, Swerdloff RS, Bray GA, Dahms WT, Atkinson RL. Low serum testosterone and sex-hormone-binding-globulin in massively obese men. J Clin Endocrinol Metab 1977; 45: 1211-1219.

36. Matsuo K, Akahoshi M, Nakashima E, Seto S, Yano K. Clinical characteristics of subjects with the Brugada-type electrocardiogram: A case control study. J Cardiovasc Electrophysiol 2004; 15: 653-657.

37. Shimizu W, Matsuo K, Kokubo Y, Satomi K, Kurita T, Noda T, et al. Sex hormone and gender difference--role of testosterone on male predominance in Brugada syndrome. J Cardiovasc Electrophysiol 2007; 18: 415-421.

38. Vatta M, Dumaine R, Varghese G, Richard TA, Shimizu W, Aihara $\mathrm{N}$, et al. Genetic and biophysical basis of sudden unexplained nocturnal death syndrome (SUNDS), a disease allelic to Brugada syndrome. Hum Mol Genet 2002; 11: 337-345.

39. Splawski I, Timothy KW, Tateyama M, Clancy CE, Malhotra A, Beggs AH, et al. Variant of SCN5A sodium channel implicated in risk of cardiac arrhythmia. Science 2002; 297: 1333-1336.

40. Pfeufer A, Jalilzadeh S, Perz S, Mueller JC, Hinterseer M, Illig T, et al. Common variants in myocardial ion channel genes modify the QT interval in the general population: Results from the KORA study. Circ Res 2005; 96: 693-701.

41. Bezzina CR, Shimizu W, Yang P, Koopmann TT, Tanck MWT, Miyamoto Y, et al. A common sodium channel promoter haplotype in Asian subjects underlies variability in cardiac conduction. Circulation 2006; 113: 338-344. 